\title{
Atomic gas far away from the Virgo cluster core galaxy NGC 4388
}

\section{A possible link to isolated star formation in the Virgo cluster?}

\author{
B. Vollmer and W. Huchtmeier
}

Max-Planck-Institut für Radioastronomie, Auf dem Hügel 69, 53121 Bonn, Germany

Received 12 February 2003 / Accepted 3 April 2003

\begin{abstract}
We have discovered $6 \times 10^{7} M_{\odot}$ of atomic gas at a projected distance greater than $4^{\prime}(20 \mathrm{kpc})$ from the highly inclined Virgo spiral galaxy NGC 4388. This gas is most probably connected to the very extended H $\alpha$ plume detected by Yoshida et al. (2002). Its mass makes a nuclear outflow and its radial velocity a minor merger as the origin of the atomic and ionized gas very unlikely. A numerical ram pressure simulation can account for the observed HI spectrum and the morphology of the H $\alpha$ plume. An additional outflow mechanism is still needed to reproduce the velocity field of the inner $\mathrm{H} \alpha$ plume. The extraplanar compact HII region recently found by Gerhard et al. (2002) can be explained as a stripped gas cloud that collapsed and decoupled from the ram pressure wind due to its increased surface density. The star-forming cloud is now falling back onto the galaxy.
\end{abstract}

Key words. galaxies: individual: NGC 4388 - galaxies: interactions - galaxies: ISM - galaxies: kinematics and dynamics

\section{Introduction}

The Virgo cluster spiral galaxy NGC 4388 is located at a projected distance of $1.3^{\circ}$ from the Virgo cluster center (M87). Its high radial velocity of $\sim 1400 \mathrm{~km} \mathrm{~s}^{-1}$ with respect to the cluster mean and its estimated line-of-sight distance (Tully-Fisher method; Yasuda et al. 1997) to the cluster center place its three dimensional location very close to M87. In addition, NGC 4388 represents one of the nearest Seyfert 2 galaxies and the first AGN to be found in the Virgo cluster (Phillips $\&$ Malin 1982). Veilleux et al. (1999) made observations with a Fabry-Perot Interferometer in the $\mathrm{H} \alpha$ and $\mathrm{O}[\mathrm{III}] \lambda 5007$ lines. They found a large, high-ionization plume extending northeastwards from the nucleus up to a projected distance of $4 \mathrm{kpc}^{1}$ above the plane of the galaxy. The plume is blueshifted with respect to the galaxy's systemic velocity. Yoshida et al. (2002) discovered a very large $\mathrm{H} \alpha$ plume that extends up to $\sim 35 \mathrm{kpc}$ northeastwards. This region contains $\sim 10^{5} M_{\odot}$ of ionized gas. They argued that the dominating source of ionization is the radiation coming from the nucleus. Veilleux et al. (1999) discussed 4 different origins for the $4 \mathrm{kpc}$ plume: (i) a minor merger, (ii) a galactic wind, (iii) nuclear outflow, and (iv) ram pressure stripping. Based on their data they favoured a combination of (iii) and (iv). Yoshida et al. (2002) on the other hand favoured scenario (i) and (iv). A surprisingly new result is that Gerhard et al. (2002) found an isolated compact HII region at a projected distance of $17 \mathrm{kpc}$ north and $4.4 \mathrm{kpc}$ west

Send offprint requests to: $\mathrm{B}$. Vollmer,

e-mail: bvollmer@mpifr-bonn.mpg.de

${ }^{1}$ We adopt a distance of $17 \mathrm{Mpc}$ to the Virgo cluster. of the galaxy center. The age of the dozen $\mathrm{O}$ stars embedded in this region ( $\sim 3 \mathrm{Myr}$ ) implies that they have formed far outside the main star-forming regions of the galaxy and perhaps even within the hot intracluster medium. Gerhard et al. (2002) argued that a radial velocity of $150 \mathrm{~km} \mathrm{~s}^{-1}$ excludes that the involved gas has been pushed to this large distance by ram pressure stripping. In addition to the studied isolated compact HII region they found 17 HII region candidates in the data of Yoshida et al. (2002). In this article we report the detection of atomic gas at a projected distance of more than $20 \mathrm{kpc}$ northeastwards from the center of NGC 4388. The observations and their results are described in Sects. 2 and 3. The dynamical model is presented in Sect. 4. We compare a model snapshot with our observations and discuss possible implications in Sect. 5. The conclusions are given in Sect. 6.

\section{Observations}

On March, 14-21 2002 we performed 21-cm line observations at 5 different positions at a frequency corresponding to the systemic velocity of NGC 4388 with a bandwidth of $12.5 \mathrm{MHz}$. The two channel receiver had a system noise of $\sim 30 \mathrm{~K}$. The 1024 channel autocorrelator was split into four parts with 256 channels, yielding a channel separation of $\sim 10 \mathrm{~km} \mathrm{~s}^{-1}$. We further binned the channels to obtain a final channel separation of $\sim 20 \mathrm{~km} \mathrm{~s}^{-1}$. One central position and four positions at a distance of one beamsize $\left(9.3^{\prime}\right)$ to the NW, SW, SE, and NE from the galaxy center were observed in on-off mode ( 5 min on source, 5 min off source). The integration times are shown in Table 1. Care was taken to avoid other Virgo galaxies with velocities within our bandwidth in all 5 on and off source 
Table 1. Integration times and rms.

\begin{tabular}{cccccc}
\hline \hline Position & Center & Northwest & Southwest & Southeast & Northeast \\
\hline$\Delta t$ (min) & 190 & 80 & 120 & 120 & 350 \\
\hline rms (mJy) & 1.2 & 1.7 & 1.1 & 1.2 & 0.9 \\
\hline
\end{tabular}

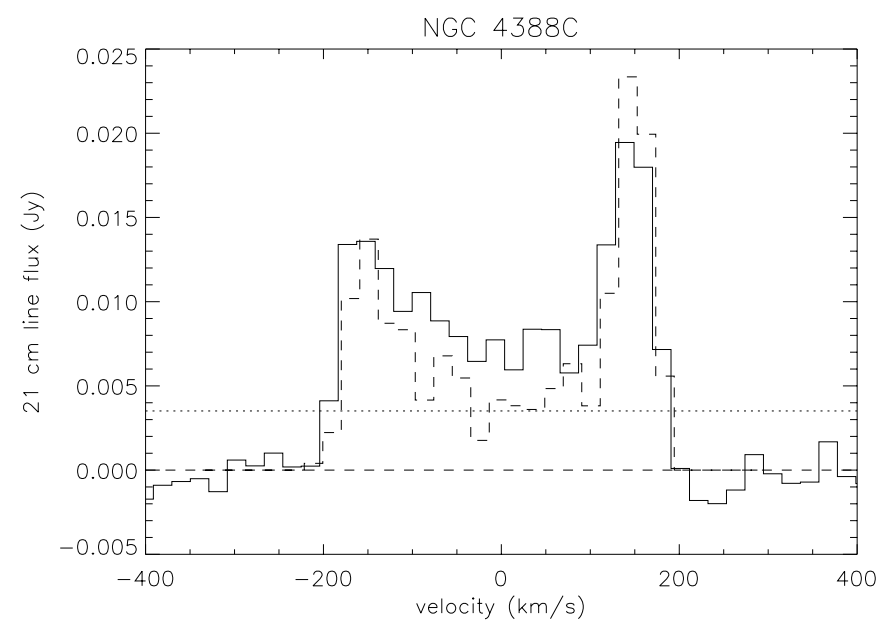

Fig. 1. Solid line: Effelsberg 100-m spectrum of the central position. Dashed line: spectrum of the VLA data. Dotted line: $3 \sigma$ noise level of the $100-\mathrm{m}$ spectrum. Heliocentric velocities are given relative to the systemic velocity of NGC $4388\left(v_{\mathrm{sys}}=2524 \mathrm{~km} \mathrm{~s}^{-1}\right)$.

positions. We used 3C286 for pointing and flux calibration. The observation times ranged between $80 \mathrm{~min}$ and $350 \mathrm{~min}$ per position. The resulting noise (Table 1 ) is partly determined by small amplitude interferences, but is close to the theoretical noise of $2 \mathrm{mJy}$ per hour of integration.

\section{Results}

The Effelsberg 100-m spectrum of the central position can be seen in Fig. 1 (solid line). It shows a double-horn structure with a $\sim 25 \%$ higher peak emission at the receding side. The total flux is $S_{\mathrm{HI}}=3.9 \mathrm{Jy} \mathrm{km} \mathrm{s}^{-1}$, which corresponds to a total HI mass of $M_{\mathrm{HI}}=2.7 \times 10^{8} M_{\odot}$.

In order to compare our off-center positions to interferometric data where the galaxy is spatially resolved, we use VLA $21 \mathrm{~cm} \mathrm{C}$ array data (Cayatte et al. 1990). These data have a spatial resolution of $23^{\prime \prime} \times 17^{\prime \prime}$ and a channel separation of $20 \mathrm{~km} \mathrm{~s}^{-1}$. We clipped the data cube at a level of $4 \mathrm{mJy} / \mathrm{beam}$ and cut out the inner $4^{\prime} \times 3^{\prime}$ around the galaxy center. The resulting spectrum for the central position is represented by the dashed line in Fig. 1. The total flux and peak flux density of Cayatte et al. (1990) are $20 \%$ higher than our values, which is within the calibration accuracy. Both spectra agree reasonably well.

For the 4 off-center positions we have synthesized spectra from the VLA data using the position of the pointing and

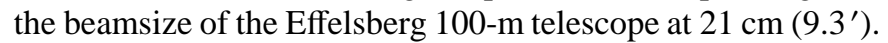
We use the spatial information of the VLA data to construct the synthesized spectra. Figure 2 shows the 100-m spectra of these positions (solid lines) together with the synthesized VLA spectra. The VLA spectra represent HI gas located in the disk (see Cayatte et al. 1990). If there is an emission excess in the offcenter 100-m spectra this must be due to gas located outside the disk. The rms noise of the north-eastern (NE) position is certainly overestimated, because of a relatively strong standing wave that had to be subtracted. We made 3 independent observations of this position in 3 different nights. In all 3 sub-spectra the signal is detected. One of them shows a flat baseline without a standing wave. The peak emission of none of the synthesized VLA spectra exceeds the 100-m 3o noise level, except the NE position where we have overestimated the rms.

Only the 100-m spectrum of the NE position shows a detection. The main peak is around zero radial velocity with respect to the galaxy. It has a width of $\sim 80 \mathrm{~km} \mathrm{~s}^{-1}$. In addition, we detect a second smaller maximum at $160 \mathrm{~km} \mathrm{~s}^{-1}$, which has the same position and width as the corresponding emission of the eastern, receding side of NGC 4388. However, it contains $\sim 30 \%$ more flux than the corresponding maximum of the VLA data. The total flux of the emission is $S_{\mathrm{HI}}=0.9 \mathrm{Jy} \mathrm{km} \mathrm{s}^{-1}$, which corresponds to an atomic gas mass of $M_{\mathrm{HI}}=6 \times 10^{7} M_{\odot}$.

\section{The model}

Since the model is described in detail in Vollmer et al. (2001), we summarize only its main features. The N-body code consists of two components, a non-collisional component that simulates the stellar bulge/disk and the dark halo, and a collisional component that simulates the ISM.

The 20000 particles of the collisional component represent gas cloud complexes which are evolving in the gravitational potential of the galaxy.

The total gas mass is $M_{\text {gas }}^{\text {tot }}=3.8 \times 10^{9} M_{\odot}$, which corresponds to the total neutral gas mass before stripping assuming an HI deficiency of 1.1. To each particle a radius is attributed depending on its mass. During the disk evolution the particles can have inelastic collisions, the outcome of which (coalescence, mass exchange, or fragmentation) is simplified following Wiegel (1994). This results in an effective gas viscosity in the disk.

As the galaxy moves through the ICM, its clouds are accelerated by ram pressure. Within the galaxy's inertial system its clouds are exposed to a wind coming from the opposite direction of the galaxy's motion through the ICM. The temporal ram pressure profile has the form of a Lorentzian, which is realistic for galaxies on highly eccentric orbits within the Virgo cluster (Vollmer et al. 2001). The effect of ram pressure on the clouds is simulated by an additional force on the clouds in the wind direction. Only clouds which are not protected by other clouds against the wind are affected.

The non-collisional component consists of 49125 particles, which simulate the galactic halo, bulge, and disk. The characteristics of the different galactic components are shown in Table 2. The resulting rotation velocity is $\sim 200 \mathrm{~km} \mathrm{~s}^{-1}$ and the rotation curve is flat. 

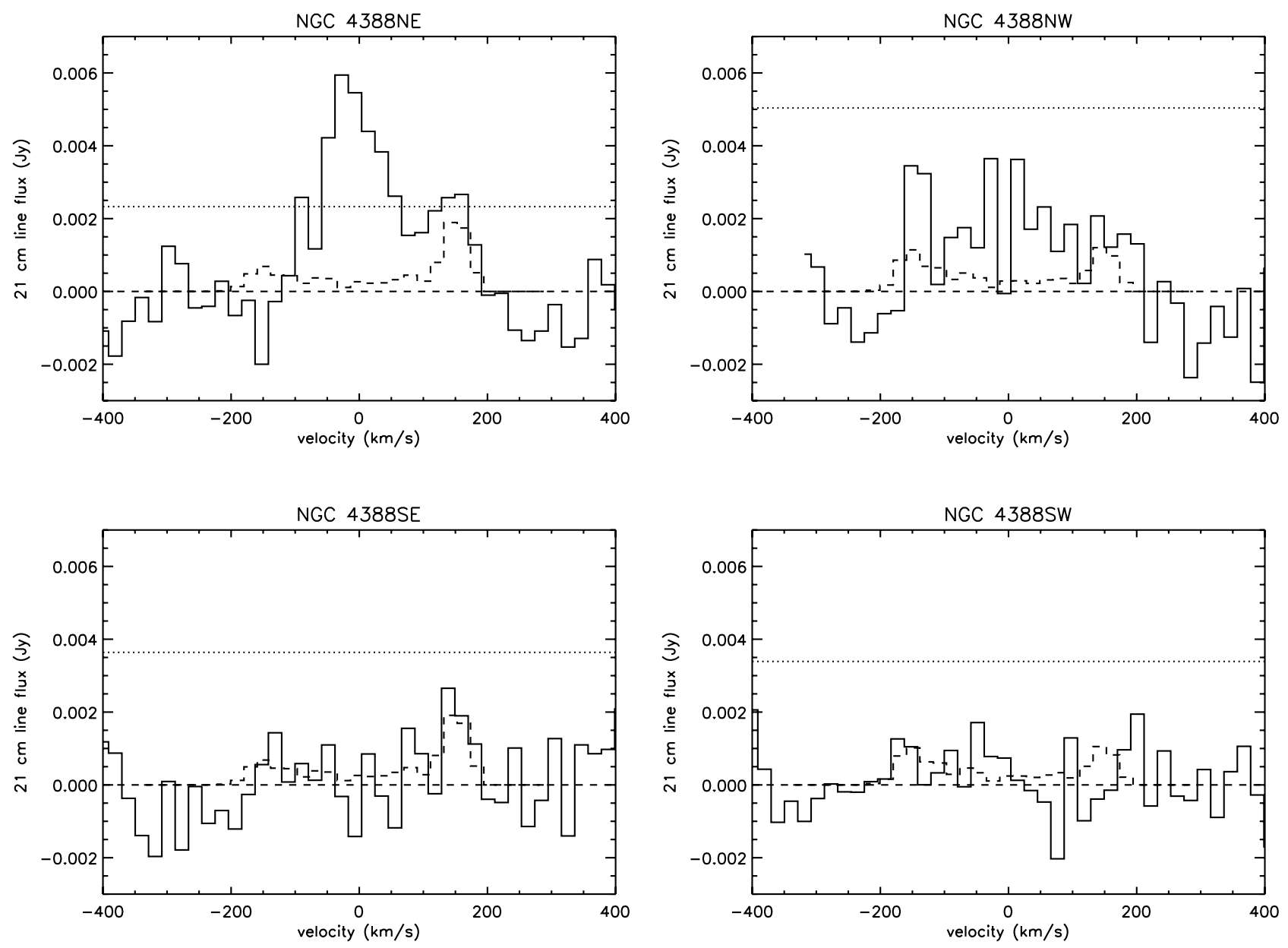

Fig. 2. Solid lines: Effelsberg 100-m spectra of the four off-center positions. Their locations with respect to the galaxy center are marked on top of each panel. Dashed line: synthesized VLA spectra which only show HI disk emission. Dotted line: $3 \sigma$ noise levels of the 100-m spectra. Velocities are given relative to the systemic velocity of NGC 4388.

Table 2. Total mass, number of particles $N$, particle mass $M$, and smoothing length $l$ for the different galactic components.

\begin{tabular}{lllll}
\hline \hline Component & $M_{\mathrm{tot}}\left(M_{\odot}\right)$ & $N$ & $M\left(M_{\odot}\right)$ & $l(\mathrm{pc})$ \\
\hline Halo & $2.4 \times 10^{11}$ & 16384 & $1.4 \times 10^{7}$ & 1200 \\
Bulge & $8.2 \times 10^{9}$ & 16384 & $5.0 \times 10^{5}$ & 180 \\
Disk & $4.1 \times 10^{10}$ & 16384 & $2.5 \times 10^{6}$ & 240 \\
\hline
\end{tabular}

The particle trajectories are integrated using an adaptive timestep for each particle. This method is described in Springel et al. (2001). The following criterion for an individual timestep is applied:

$\Delta t_{\mathrm{i}}=\frac{20 \mathrm{~km} \mathrm{~s}^{-1}}{a_{\mathrm{i}}}$,

where $a_{\mathrm{i}}$ is the acceleration of the particle "i". The minimum of all $t_{\mathrm{i}}$ defines the global timestep used for the Burlisch-Stoer integrator that integrates the collisional component.

The galaxy is on an eccentric orbit within the cluster. The temporal ram pressure profile can be described by:

$p_{\text {ram }}=\frac{p_{\text {max }}}{t^{2}+t_{\mathrm{HW}}^{2}}$, where $t_{\mathrm{HW}}$ is the width of the profile (Vollmer et al. 2001). We set $p_{\max }=5000 \mathrm{~cm}^{-3}\left(\mathrm{~km} \mathrm{~s}^{-1}\right)^{2}$ and $t_{\mathrm{HW}}=50 \mathrm{Myr}$. The efficiency of ram pressure also depends on the inclination angle $i$ between the galactic disk and the orbital plane (Vollmer et al. 2001). We set $i=45^{\circ}$.

Figures 3 and 4 show the evolution of the stellar and gas disk during the galaxy's passage through the cluster core. The galaxy is seen face-on in Fig. 3. In Fig. 4 the position and inclination angle of NGC $4388\left(\mathrm{PA}=90^{\circ}, i=85^{\circ}\right)$ are used. The time of closest approach to the cluster center, i.e. maximum ram pressure, is $t=0 \mathrm{Myr}$. Since only the gas is affected by ram pressure, the stellar disk does not change during the whole simulation. At $t \sim-80 \mathrm{Myr}$ the external $\sim 5 \mathrm{kpc}$ ring of neutral gas begins to be pushed to the north (Fig. 4). At maximum ram pressure $(t=0 \mathrm{Myr})$ this northern extraplanar gas shows 2 distinct features: (i) an approximately uniform gas layer in the north of the disk and (ii) a horizontal gas ridge located to the north east. This is where the accelerated gas, which was initially rotating against the direction of the galaxy's velocity (at $(0 \mathrm{kpc}, 20 \mathrm{kpc})$ in Fig. 3) and already rotated $\sim 180^{\circ}$, is now again compressed by ram pressure (at $(-20 \mathrm{kpc}, 0 \mathrm{kpc})$ in Fig. 3). These structures expand in the following evolution of the neutral gas. For $t>40 \mathrm{Myr}$ a counter-rotating arm is 

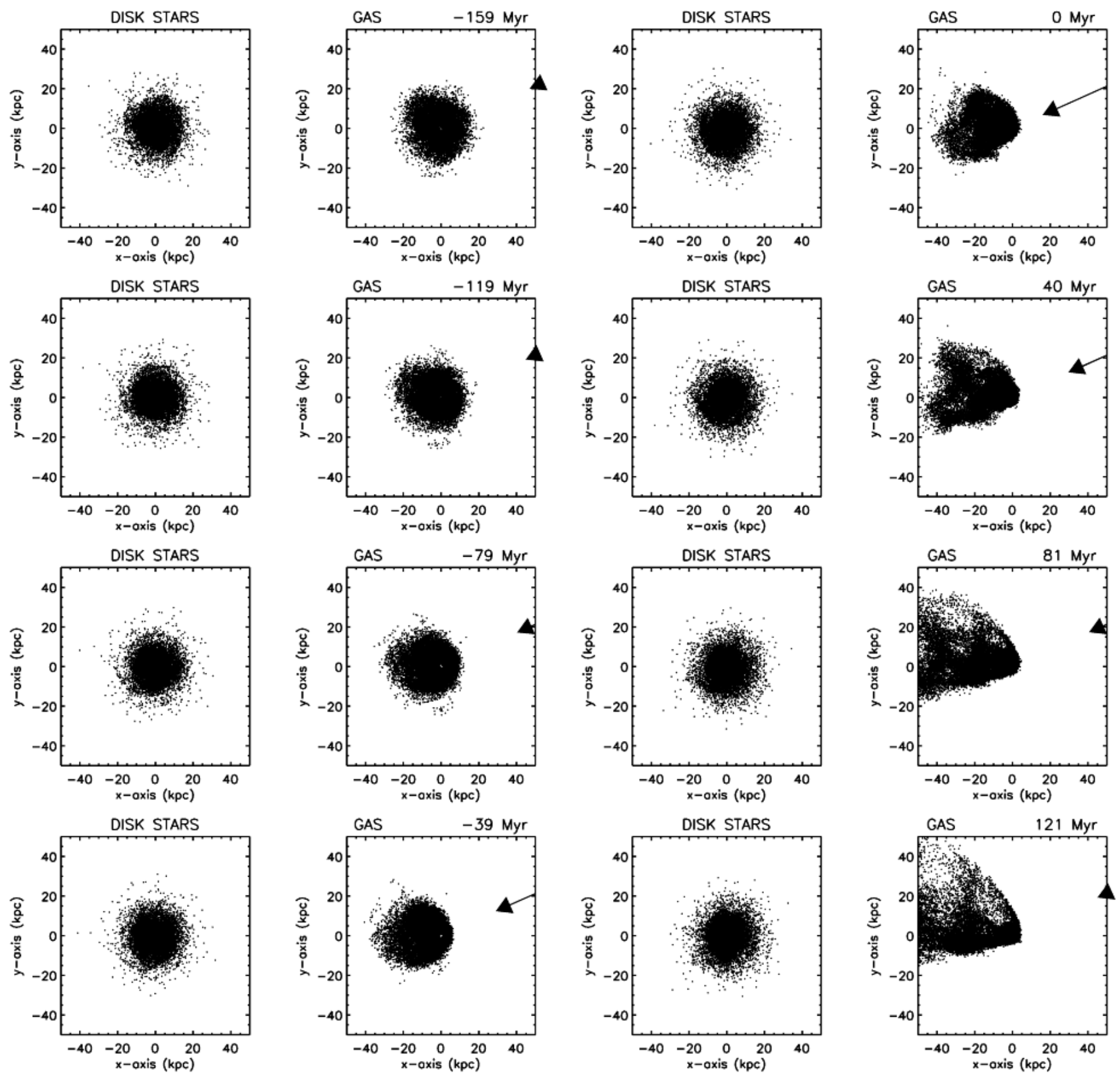

Fig. 3. Evolution of the model stellar and gas disk. The disk is seen face-on and rotates counter-clockwise. The arrow indicates the direction of ram pressure, i.e. it is opposite to the galaxy's velocity vector. The size of the arrow is proportional to $\rho v_{\mathrm{gal}}^{2}$. The galaxy passes the cluster core at 0 Myr. The time to the core passage is marked at the top of the panels. (An animation of the temporal evolution is available at http://www.edpsciences.org).

forming (see Vollmer et al. 2001), which is responsible for the diagonal gas feature in the south-west (Fig. 4).

In order to show the effect of ram pressure on the radial velocities, we show the latter as a function of the projected distance from the galaxy center for the last snapshot $(t \sim 120 \mathrm{Myr})$ in Fig. 5. From 0 to $30 \mathrm{kpc}$ the radial velocities with respect to the galaxy center show an almost constant gradient from $100 \mathrm{~km} \mathrm{~s}^{-1}$ to $-50 \mathrm{~km} \mathrm{~s}^{-1}$. At a distance of $\sim 30 \mathrm{kpc}$ the velocities bifurcate. One part continues with the same gradient, the other part shows a much steeper gradient. The minimum velocities are $-600 \mathrm{~km} \mathrm{~s}^{-1}$. The minimum absolute velocities are $|v| \sim 800 \mathrm{~km} \mathrm{~s}^{-1}$. The main gas mass is found at a projected distances between $20 \mathrm{kpc}$ and $30 \mathrm{kpc}$ and radial velocities between $-50 \mathrm{~km} \mathrm{~s}^{-1}$ and $0 \mathrm{~km} \mathrm{~s}^{-1}$. At this timestep, the galaxy has an absolute velocity of $\sim 2000 \mathrm{~km} \mathrm{~s}^{-1}$ with respect to the cluster mean velocity. From $p_{\max }=5000 \mathrm{~cm}^{-3}\left(\mathrm{~km} \mathrm{~s}^{-1}\right)^{2}$ we derive a maximum ICM density of $n_{\mathrm{ICM}}^{\max } \sim 3 \times 10^{-3} \mathrm{~cm}^{-3}$. Using the ICM density profile of Schindler et al. (1999), this corresponds to an impact parameter of $b \sim 100 \mathrm{kpc}$, which is consistent with 

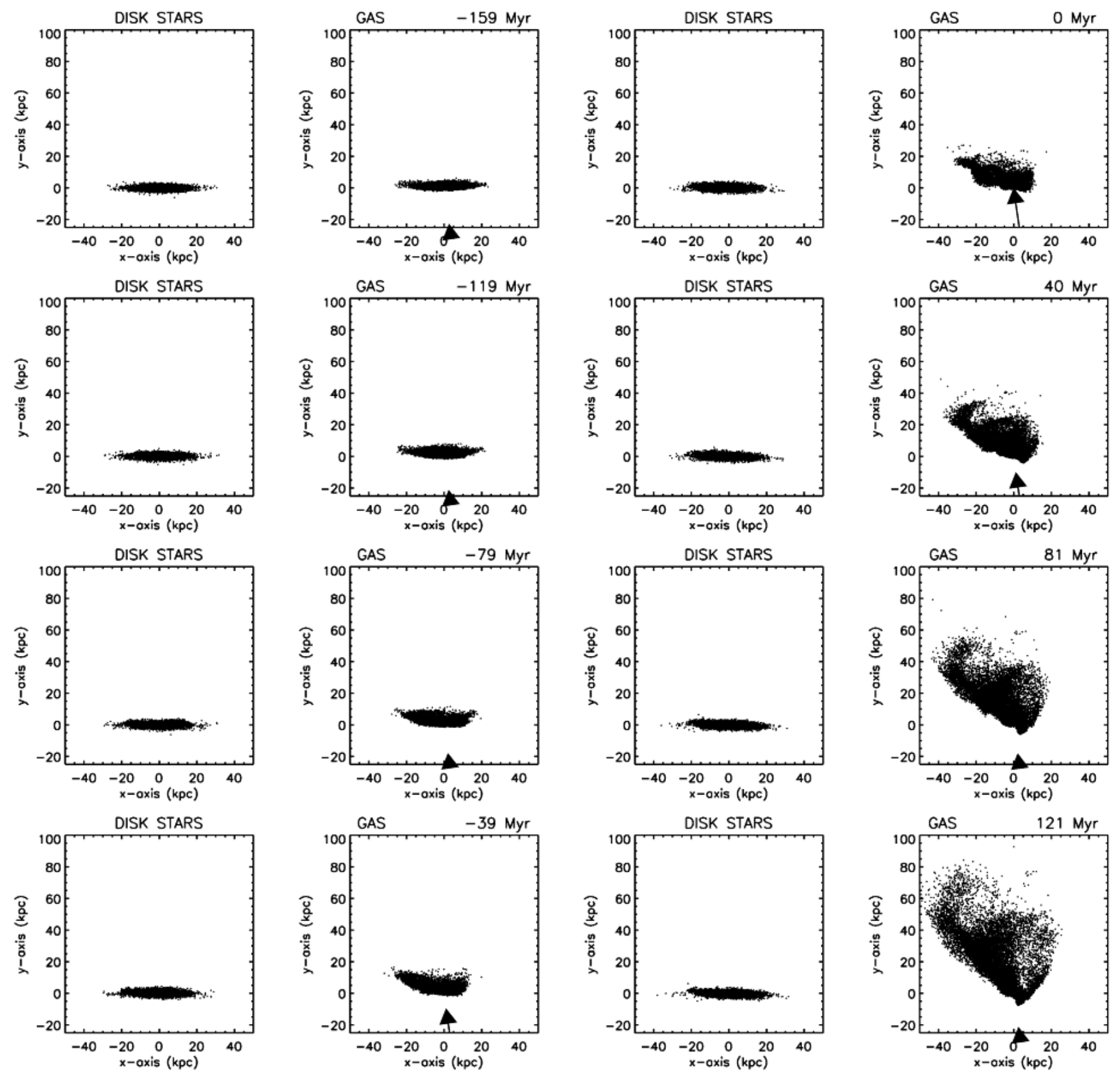

Fig. 4. Evolution of the model stellar and gas disk. The position and inclination angle of NGC $4388\left(\mathrm{PA}=90^{\circ}, i=85^{\circ}\right)$ are used. The arrow indicates the direction of ram pressure, i.e. it is opposite to the galaxy's velocity vector. The size of the arrow is proportional to $\rho v_{\mathrm{gal}}^{2}$. The galaxy passes the cluster core at $0 \mathrm{Myr}$. The time to the core passage is marked at the top of the panels. (An animation of the temporal evolution is available at http://www. edpsciences.org).

the dynamical model of Vollmer et al. (2001). We compare the final timestep $(t \sim 120 \mathrm{Myr})$ with our observations.

\section{Discussion}

Despite the fact that the $100-\mathrm{m}$ spectra do not give precise informations about the location of the detected HI gas, we can conclude from our data that the component around zero radial velocity lies at a distance of at least half a beamsize $\left(4.7^{\prime}\right.$ or $23 \mathrm{kpc}$ ) away from the galaxy center. It contains several $10^{7}$ solar masses. If it was closer to the galaxy one should see it in the VLA synthesized spectrum if it is high column density gas or alternatively in the central 100-m spectrum if it was low column density, extended gas. We suggest that it is connected to the very extended $\mathrm{H} \alpha$ plume discovered by Yoshida et al. (2002).

To our knowledge this is the first time that atomic gas is observed at these large distances from a galaxy in a cluster core region. What is the origin of this extraplanar gas?

Veilleux et al. (1999) discussed 4 different mechanisms (cf. Sect. 1) and favoured a mixture of nuclear outflow and ram pressure stripping. Yoshida et al. (2002) prefered a minor 


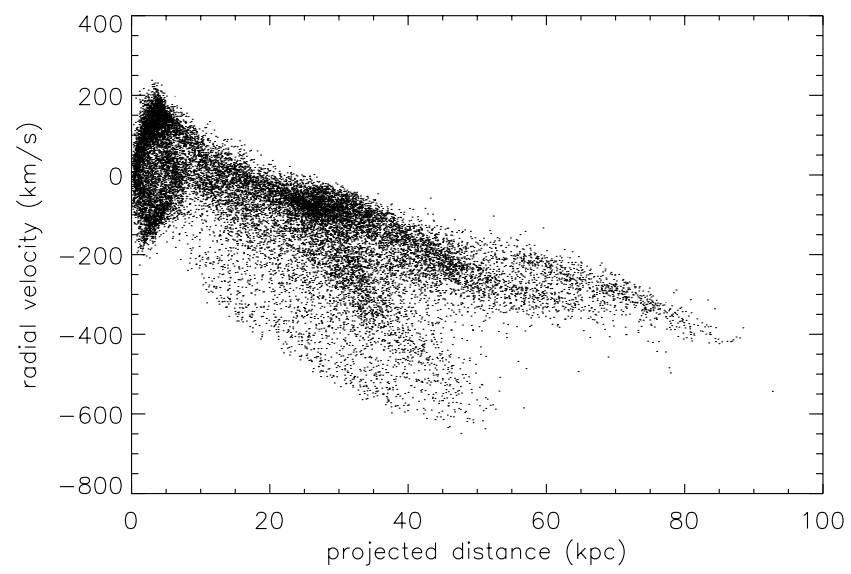

Fig. 5. ISM radial velocity as a function of the projected distance to the galaxy center.

merger and/or ram pressure stripping. If the detected atomic gas is connected to the very extended $\mathrm{H} \alpha$ plume, our data rule out a nuclear outflow as the origin of this plume, because of its large total gas mass. A superwind that has recently ceased could have provided enough energy (see e.g. Heckman et al. 1990), but there is no indication for a recent, strong starburst in the center of NGC 4388. If the atomic gas and the very extended $\mathrm{H} \alpha$ plume represent the debris of a minor merger, its gas velocity around relative zero places its orbit in the plane of the sky. In our understanding this is inconsistent with the morphology of the $\mathrm{H} \alpha$ plume. If the $\mathrm{H} \alpha$ emission represents the debris of a tidally disrupted dwarf galaxy, it should trace its trajectory. In this picture the curvature of the north-eastern part of the very extended $\mathrm{H} \alpha$ emission is dynamically difficult to explain. In addition, the $\mathrm{H} \alpha$ plume is larger close to the disk, which is also in contradiction to the tidal debris scenario.

We suggest a model, where the atomic gas and the $\mathrm{H} \alpha$ are connected and both represent ram pressure stripped gas in different phases. In Fig. 6 (left panel) we show a possible ram pressure stripping scenario. The galaxy moves to the south and away from the observer. It is on a radial orbit and its core passage is $\sim 120 \mathrm{Myr}$ ago (Sect. 4). The galaxy has lost $\sim 90 \%$ of its atomic gas. We show only gas with a local density greater than $n \sim 30 \mathrm{~cm}^{-3}$. For clarity the greyscales are inverted, i.e. dark regions correspond to low column density gas. Since the stars are not affected by ram pressure, the stellar disk is symmetric. The gas disk is strongly truncated at a radius of $\sim 5 \mathrm{kpc}\left(\sim 1^{\prime}\right)$. This is in agreement with the VLA observations of Cayatte et al. (1990). Only the high density eastern edge of the stripped, extraplanar gas distribution can be seen. In addition, the counter-rotating, backfalling arm in the southwest has high local densities and thus appears in Fig. 6 (left panel). The main off-plane gas mass is located at $(-20 \mathrm{kpc}$, $20 \mathrm{kpc}$ ). For simulations with smaller maximum ram pressure, it is located closer to the galaxy. The corresponding velocity field is shown in Fig. 6 (right panel). Within the disk plane, the velocity field is dominated by rotation. The western side of the disk is approaching the observer. The stripped gas at distances smaller than $10 \mathrm{kpc}$ above the disk plane has positive velocities, whereas the gas at distances greater than $10 \mathrm{kpc}$ has negative velocities. The counter-rotating, backfalling arm has a mean radial velocity of $\sim 0 \mathrm{~km} \mathrm{~s}^{-1}$.

Figure 7 shows the model $100-\mathrm{m}$ spectrum at central position (left panel) and the northeastern (NE) position (right panel). We find a total $\mathrm{HI}$ mass of $\sim 5 \times 10^{8} M_{\odot}$ and $\sim 7 \times 10^{7} M_{\odot}$ at the central and north-eastern position, respectively.

The central spectrum shows a double horn structure and the receding side has a higher peak flux as it is observed (Fig. 1). However, a part of the flux from the high velocity end of the approaching side is missing, mainly because it has a lower local density.

For the north-eastern position we observe a clear resemblance between the model and the observed spectrum (Fig. 2). The maximum at $160 \mathrm{~km} \mathrm{~s}^{-1}$ is due to the extended eastern, receding side, whereas the main maximum around $\sim-50 \mathrm{~km} \mathrm{~s}^{-1}$ radial velocity is due to the material at distances of about $20 \mathrm{kpc}$ from the galactic disk (Fig. 6). Thus the ram pressure scenario can account for the very extended tail. However, it is not possible to reproduce the velocity field of the NE $\mathrm{H} \alpha$ plume (Veilleux et al. 1999), since it shows a blueshift with respect to the galaxy's systemic velocity. In order to explain this feature an additional outflow mechanism is needed.

How can this gas survive within such a hostile environment like the intracluster medium (ICM)? Our model is not able to investigate this subject, because it considers only the neutral gas phase and does not include ISM/ICM shocks that might heat the gas. Evaporation and ionization physics are also not included. Thus we have to rely on analytical arguments.

Veilleux et al. (1999) argued that in the case classical thermal heat conduction a typical HI cloud evaporates in the hot ICM within $10^{7} \mathrm{yr}$. On the other hand, Vollmer et al. (2001) showed that within the ICM evaporation is saturated. If the stripped atomic gas clouds have column densities of $10^{21} \mathrm{~cm}^{-2}$, the evaporation time can reach $\sim 10^{8} \mathrm{yr}$. They also discussed the possibility that the dense part of the stripped neutral gas contracts due to its selfgravity, because the internal heating (stellar radiation and supernova shocks) are absent. Since the dust is stripped together with the gas, molecules might form when the gas density becomes high enough. The only gas heating is then the X-ray radiation from the hot ICM. An equilibrium cloud has a mass of several thousand solar masses, a radius of $\sim 10 \mathrm{pc}$, and a column density of a few $10^{21} \mathrm{~cm}^{-2}$. Only $10 \%$ of this column density is in form of neutral hydrogen. Could this be the case for NGC 4388? These clouds can in principle survive long enough to be pushed to the observed large distances. It is then not excluded that these clouds collapse and form stars.

Interestingly, Gerhard et al. (2002) found a compact HII region that has formed stars in situ, far away from the main starforming disk. This HII region is located at a projected distance of $17.5 \mathrm{Mpc}$ from the galaxy center in the northwest of the galactic center and has a radial velocity of $150 \mathrm{~km} \mathrm{~s}^{-1}$. Within our model it is possible that this HII region is falling back to the galaxy after having been pushed by ram pressure to a large galactocentric distance. When a stripped cloud collapses, its column density increases rapidly and it decouples from the wind (ram pressure). It then begins to fall back to the galaxy due to the galaxy's gravitational potential. With the relatively low metallicity (Gerhard et al. 2002) this stripped gas was lo- 

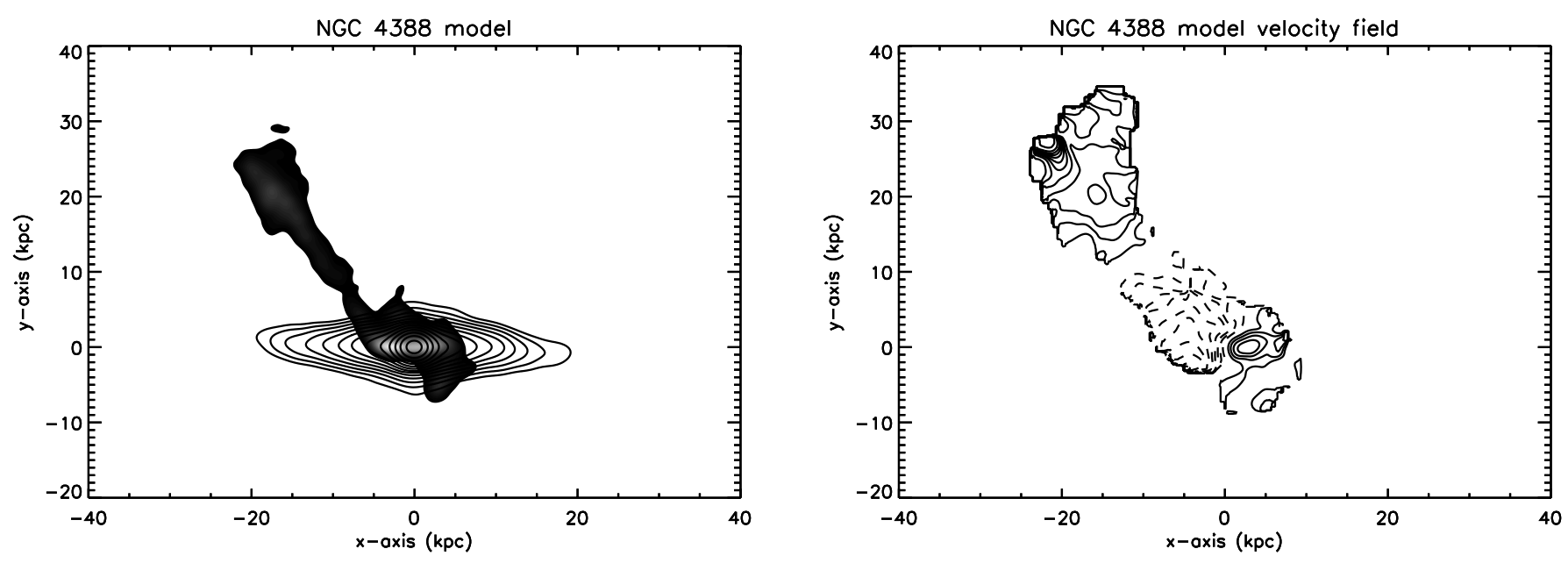

Fig. 6. Left panel: stellar distribution as contours, gas distribution as greyscales. Only gas with a local density greater than $\sim 30 \mathrm{~cm}^{-3}$ is shown. For clarity the greyscales are inverted, i.e. dark regions correspond to low column density gas. Right panel: model velocity field. Dashed lines mark positive, solid lines negative velocity contours. The velocities range between $-200 \mathrm{~km} \mathrm{~s}^{-1}$ and $260 \mathrm{~km} \mathrm{~s}^{-1}$. The contour stepsize is $20 \mathrm{~km} \mathrm{~s}^{-1}$.
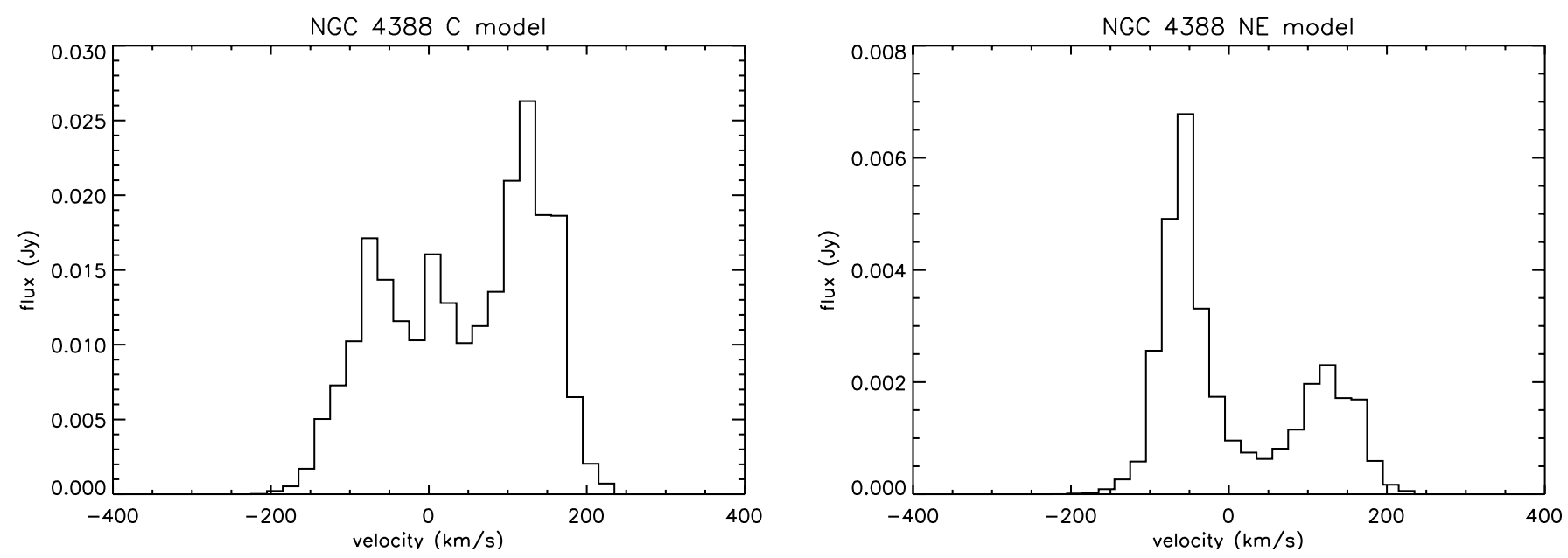

Fig. 7. Left panel: model 100-m spectrum at the central (C) position. Right panel: model 100-m spectrum at the northeastern (NE) position. Velocities are given relative to the systemic velocity of NGC 4388.

cated approximately at the optical radius $\left(R_{25}\right)$ of NGC 4388 before stripping has taken place (Duc et al. 2000). This scenario is in agreement with the positive radial velocity of the compact HII region. In addition, Gerhard et al. (2002) found a number of HII region candidates in the very extended $\mathrm{H} \alpha$ plume. Within our picture this would mean that the stripped gas clouds have become molecular and are now forming stars. This mechanism would be analog to the formation of globular clusters in the tidal tails of gravitationally perturbed galaxies.

\section{Conclusions}

We have observed the Virgo cluster core galaxy NGC 4388 in the $21 \mathrm{~cm}$ HI line with the Effelsberg 100-m telescope at 5 different positions: one in the center and 4 located one $100-\mathrm{m}$ beamsize to the NE, NW, SW, and SE from the galaxy center. The results of these observations are compared to a realistic dynamical model including ram pressure stripping. The results are:
1. We detected extraplanar HI gas of $\sim 6 \times 10^{7} M_{\odot}$ located at a distance of at least $20 \mathrm{kpc}$ to the north-east of the galaxy center. This gas is most probably connected to the very extended $\mathrm{H} \alpha$ plume detected by Yoshida et al. (2002).

2. The dynamical model is able to reproduce the HI deficiency, the truncated HI disk and the extraplanar emission. NGC 4388 passed the cluster core $\sim 120$ Myr ago.

3. Within this scenario the maximum ram pressure is $p_{\max }=$ $5000 \mathrm{~cm}^{-3}\left(\mathrm{~km} \mathrm{~s}^{-1}\right)^{-2}$, the maximum ICM density encountered during core passage is $n_{\mathrm{ICM}} \sim 3 \times 10^{-3} \mathrm{~cm}^{-3}$, the galaxy's absolute velocity with respect to the cluster mean is $\sim 2000 \mathrm{~km} \mathrm{~s}^{-1}$, and the angle between the disk and the orbital plane is $i=45^{\circ}$.

4. The extraplanar compact HII region recently found by Gerhard et al. (2002) can be explained as a stripped gas cloud that collapsed and decoupled from the ram pressure wind due to its increased surface density.

Acknowledgements. Based on observations with the 100-m telescope of the MPIfR (Max-Planck-Institut für Radioastronomie) at 
Effelsberg. The authors would like to thank V. Cayatte and J. Schindler, S., Binggeli, B., \& Böhringer, H. 1999, A\&A, 343, 420 van Gorkom for providing us kindly their VLA data.

Springel, V., Yoshida, N., \& White, D. M. 2001, NA, 6, 79

Veilleux, S., Bland-Hawthorn, J., Cecil, G., Tully, B. R., \& Miller, S. 1999, ApJ, 520, 111

\section{References}

Cayatte, V., van Gorkom, J. H., Balkowski, C., \& Kotanyi, C. 1990, AJ, 100, 604

Vollmer, B., Cayatte, V., Balkowski, C., \& Duschl, W. J. 2001, ApJ, 561,708

Vollmer, B. 2003, A\&A, 398, 525

Cayatte, V., Kotanyi, C., Balkowski, C., \& van Gorkom, J. H. 1994, AJ, 107, 1003

Duc, P.-A., Brinks, E., Springel, V., et al. 2000, AJ, 120, 1238

Wiegel, W. 1994, Diploma Thesis, University of Heidelberg

Yasuda, N., Fukugita, M., \& Okamura, S. 1997, ApJS, 108, 417

Yoshida, M., Yagi, M., Okamura, S., et al. 2002, ApJ, 567, 118

Gerhard, O., Arnaboldi, M., Freeman, K. C., \& Okamura, S. 2002, ApJ, 580, L121

Heckman, T. M., Armus, L., \& Miley, G. K. 1990, ApJS, 74, 833

Phillips, M. M., \& Malin, D. F. 1982, MNRAS, 199, 205 